\title{
The Feynman Propagator for Quantum Gravity: Spin Foams, Proper Time, Orientation, Causality and Timeless-Ordering
}

\author{
D. Oriti \\ Department of Applied Mathematics and Theoretical Physics, \\ Centre for Mathematical Sciences, University of Cambridge, Wilberforce Road, Cambridge CB3 OWA, UK, EU
}

Received on 19 December, 2004

\begin{abstract}
We discuss the notion of causality in Quantum Gravity in the context of sum-over-histories approaches, in the absence therefore of any background time parameter. In the spin foam formulation of Quantum Gravity, we identify the appropriate causal structure in the orientation of the spin foam 2-complex and the data that characterize it; we construct a generalised version of spin foam models introducing an extra variable with the interpretation of proper time and show that different ranges of integration for this proper time give two separate classes of spin foam models: one corresponds to the spin foam models currently studied, that are independent of the underlying orientation/causal structure and are therefore interpreted as a-causal transition amplitudes; the second corresponds to a general definition of causal or orientation dependent spin foam models, interpreted as causal transition amplitudes or as the Quantum Gravity analogue of the Feynman propagator of field theory, implying a notion of "timeless ordering".
\end{abstract}

\section{Introduction}

This paper discusses the issue of causality in non-perturbative quantum gravity, and more precisely in the spin foam formulation of the theory. This has implications for the broader issue of time in a background independent quantum theory as well as rather more technical aspects related to the specific approach we deal with. We will try to highlight both these conceptual issues and the ideas on which our results are based and what these results actually are. These results were presented in [1], and their presentation in section 3, 4 and 5 is in fact based on [1], and will be analysed and discussed in more details in [2].

\subsection{Time and causality in Quantum Gravity}

In quantum gravity, because of background independence and diffeomorphism invariance, neither phsyical observables nor physical states, i.e. none of the basic elements of any canonical formulation of the theory, can depend on an external time parameter as is the case in ordinary quantum mechanics, but time itself becomes a quantum variable insofar as the metric itself is a quantum variable; this is true also in a covariant or sum-over-histories formulation, because, even if the boundary may be assigned timelike data, still the spacetime geometry cannot be used to define any unique notion of time in the interior of the manifold as it represents the quantum field we are summing over to define our theory. This led to the conclusion that time can not be a basic ingredient in a proper formulation of Quantum Gravity, or that the very concept of time is not fundamantal at all and should instead emerge only in a semiclassical approximation [3]. Regarding the issue of causality, there seem to be two basic attitude one can take: one can hold that we cannot speak of causality in absence of time and therefore just as time itself also a notion causality should emerge and be applicable only in a semiclassical limit; this is actually true without argument, actually a truism, if we stick to the conventional notion of causality in terms of a given spacetime geometry, and of lightcone structures in a continuous manifold; however one can argue that the notion of causality is actually more primitive than that of time, more fundamental, being already present in the notion of ordering between events, of a fundamental directionality in spacetime, and that as such can be present, as a seed of what will then be, in a semiclassical approximation, the usual notion of causality, even if discrete or combinatorial or algebraic structures are used to encode gravitational degrees of freedom and thus spacetime geometry in a formulation of Quantum Gravity. We agree with this latter point of view.

\subsection{Causality as ordering in Quantum Gravity}

Indeed, certain approaches to Quantum Gravity even take this notion of causality as order as the basic ingredient for their constructions, the keystone on which to found the theory; it is the case of the causal set approach [4] where spacetime at the most fundamental level is taken to be a set of fundamental events endowed with an ordering representing their causal relations and the task is that of constructing a suitable quantum amplitude for each configuration so specified, this amplitude being the main ingredient ina sum-over-histories formulation of quantum gravity as a sum over causal sets, i.e. a sum over 
causally ordered or oriented, discrete sets of events.

This idea of causality as ordering as a basic ingredient in a sum-over-histories formulation of the theory has a formal implementation also in the analytic path integral approach to quantum gravity as developed by Teitelboim [5], so not assuming any discrete substratum for spacetime, and his construction can be seen as a formal realization of what seems to be a general principle in any sum-over-histories quantum gravity theory: implement the causality principle by requiring that each history summed over, i.e. each spacetime geometry, is oriented, that this orientation matches that of the boundary states/data and is registered in the quantum amplitude assigned to each history, such that, when a canonical formulation is available, the two boundary states can be distinguished as either 'in-state' or 'out-state'; this orientation dependence replaces the notion of 'time-ordering' of quan- tum field theory, that is of course unavailable in this background independent context. The fact that this is possible or not, thus the exact way the Quantum Gravity transition amplitudes are defined allows a distinction between different kinds of transition amplitudes, causal and a-causal ones. More precisely [5], the distinction between the different transition amplitudes in registering initial and final 3-geometry $\left(h^{1}, h^{2}\right)$ in the boundary data of the path integral is obtained by choosing different range of integration in the proper time formulation of the theory; using a canonical decomposition of the metric variables, the role of proper time is played by the lapse function; an unrestricted range of integration leads to the (quantum gravity analogue of the) Hadamard function, while a restriction to positive lapses leads to the (analogue of the) Feynman propagator. The expressions for the two quantities have just a formal meaning, but read:

$$
\begin{array}{r}
G_{H}\left(h^{2}, h^{1}\right)=\int_{-\infty}^{+\infty} \mathcal{D} N\left[e^{i \int_{\mathcal{M}} d^{3} x d t}\left(\pi^{i j} \dot{h}_{i j}-N \mathcal{H}-N^{i} \mathcal{H}_{i}\right) \mathcal{D} \pi \mathcal{D} h\right] \\
G_{F}\left(h^{2}, h^{1}\right)=\int_{0}^{+\infty} \mathcal{D} N\left[e^{i \int_{\mathcal{M}} d^{3} x d t\left(\pi^{i j} \dot{h}_{i j}-N \mathcal{H}-N^{i} \mathcal{H}_{i}\right)} \mathcal{D} \pi \mathcal{D} h\right] .
\end{array}
$$

The idea behind this construction is best understood recalling basic facts from the theory of a quantum relativistic particle (that cna be thought indeed as quantum gravity in $0+1$ dimensions. There [6] the main difference between the different transition amplitudes (and 2-point functions) one can construct, in particular the Feynman propagator and the Hadamard function, is exactly the way they encode (or fail to encode) causality restrictions, in the fact that the amplitude registers or not that one of the two is in the causal future of the other; this order can be imposed in each of the histories summed over in a clear way using a proper time formulation: starting from the same proper time dependent expression $g\left(x_{1}, x_{2}, T\right)$, which can be given a sum-over-histories form, the Hadamard function is obtained eliminating this dependence by integrating over the proper time variable with an infinite range over both positive and negative values; the resulting amplitude is then a-causal, and real, and does not register any ordering between its arguments; the Feynman propagator is instead obtained restricting this integration to positive or negative proper times only, and this ordering is precisely what makes it causal; denoting $G_{W}^{ \pm}\left(x_{1}, x_{2}\right)$ the positive and negative Wightman function, the result is:

$$
\begin{aligned}
& G_{H}\left(x_{1}, x_{2}\right)=G_{W}^{+}\left(x_{1}, x_{2}\right)+G_{W}^{-}\left(x_{1}, x_{2}\right)= \\
& =\int_{-\infty}^{+\infty} d T g\left(x_{1}, x_{2}, T\right) \\
& G_{F}\left(x_{1}, x_{2}\right)=\theta\left(x_{2}^{0}-x_{1}^{0}\right) G_{W}^{+}\left(x_{1}, x_{2}\right)+ \\
& +\theta\left(x_{1}^{0}-x_{2}^{0}\right) G_{W}^{-}\left(x_{1}, x_{2}\right)=\int_{0}^{+\infty} d T g\left(x_{1}, x_{2}, T\right) \\
& g\left(x_{1}, x_{2}, T\right)=\int d^{4} p e^{i\left[\left(x_{2}-x_{1}\right) \cdot p-T\left(p^{2}+m^{2}\right)\right]} .
\end{aligned}
$$

The problem we address in this work is how to construct the analogue quantities in a spin foam context, i.e. in a purely combinatorial, algebraic and group theoretic way, in absence of any smooth manifold structure and any metric field. We will see that this can be achieved in full generality and in a very natural way.

\section{Spin Foam models of Quantum Gravity}

Spin foam models $[7,8]$ are currently being studied as a new more rigorous implementation of the path integral approach to quantum gravity. As such they are constructed by a definition of histories of the gravitational field, interpreted as 4dimensional geometries for a given spacetime manifold, and an assignment of quantum amplitudes to these geometries, i.e. suitable complex functions of the geometric data characterizing each history. Different models have been proposed and derived from many different points of view, including lattice gauge theory type derivations [9] and group field theory formulations [10], for both the Riemannian and Lorentzian signatures, and in different dimensions, which counts as one of the attractive features of this approach. The peculiarity of the spin foam framework, as compared with the traditional path integral for gravity, is that the spacetime manifolds on which the gravitational data are given are combinatorial and discrete ones, and specifically are given by combinatorial 2-complexes, i.e. collections of vertices, edges and faces together with their relations ("what is in the boundary of what"), and the histories of the gravitational field are characterized by data taken uniquely from the representation 
theory of the local gauge group of gravity, i.e. the Lorentz group, and no familiar notions of metric on differentiable manifolds are used. While this maybe very attractive at the aesthetic/philosophical level, and turns out to be very useful at the technical level (it is because of this that precise definitions of both the measure and the amplitudes for 4geometries can be given), it makes the resulting models more difficult to interpret and to work with, as we lack all our conventional GR tools and quantities, as well as our geometric intuition. The main object that such a sum-over-histories formulation defines is the partition function of the theory, and from this, allowing the underlying spacetime manifold (2complex) to have boundaries, quantum amplitudes functions of the boundary data, that when a canonical interpretation is available should be interpreted as transition amplitudes between quantum gravity states. The partition function in these models, for a given 2-complex $\sigma$, has the general structure:

$$
Z(\sigma)=\sum_{\left\{\rho_{f}\right\}} \prod_{f} \mathcal{A}_{f}\left(\rho_{f}\right) \prod_{e} \mathcal{A}_{e}\left(\rho_{f \mid e}\right) \prod_{v} \mathcal{A}_{v}\left(\rho_{f \mid v}\right)
$$

where the amplitudes for faces $f$, edges $e$ and vertices $v$ of the 2-complex are all functions of the representations $\rho_{f}$ of the Lorentz group associated to the faces of the complex.

The analysis we are going to describe in the following concerning the role of orientation data in the spin foam context and the implementation of causality is restricted to models based on ordinary Lie groups and homogeneous spaces, like the Ponzano-Regge models [11] in 3 dimensions and the Barrett-Crane type models in 4 and higher, and not using explicitely any quantum group structure. Physically these can be interpreted as models of quantum gravity without cosmological constant. We make use of expressions for the amplitudes of the models in terms of both group variables (or variables with values in an homogeneous space) and representation variables, i.e. we use a 'first order' formulation of spin foam models [12]. We do not consider less understood spin foam models, e.g. the Reisenberger model, although it is quite likely, in our opinion, that a similar analysis can be performed also in that case. For the rest our analysis is completely general and holds in any spacetime dimension and any signature $[1,2]$. For the sake of brevity, however, we will show how the construction works more explicitely only for the Barrett-Crane type models in $n$-dimensions based on the homogeneous space $H^{n-1} \simeq S O(n-1,1) / S O(n-1)$ or $S^{n-1} \simeq S O(n) / S O(n-1)$. In these models, the amplitudes in the 1 st order formalism factorize for the different vertices of the 2-complex [7] and take the form:

$$
\begin{array}{r}
Z=\left(\prod_{f} \int d \rho_{f}\right)\left(\prod_{v} \prod_{e \in v} \int_{H_{e}} d x_{e}\right) \prod_{f} \mathcal{A}_{f}\left(\rho_{f}\right) \\
\prod_{e} \mathcal{A}_{e}\left(\rho_{f \in e}\right) \prod_{v} \mathcal{A}_{v}\left(x_{e \in v}, \rho_{f \in v}\right)
\end{array}
$$

where the 2-complex is taken to be topologically dual to a $n$ dimensional simplicial complex (with a $1-1$ correspondence between $k$-cells of the 2-complex and $n-k$-simplices of the simplicial complex) and therefore the precise combinatorics varies according to the dimension, but in any case: $\rho_{f}$ are the unitary irreps of the local gauge group of gravity $(S O)$ $1,1)$ in the n-dimensional Lorentzian case and $S O(n)$ in the n-dimensional Riemannian case), and these unitary representations are labelled by either a half-integer in the Riemannian case and in the case of discrete simple representations $(n, 0)$ of $S O(n-1,1)$, or by a real parameter in the case of continuous simple representations $(0, \rho)$ of $S O(n-1,1), H_{e}$ is the homogeneous space to which the vectors $x_{e}$ belong and is $S^{n}$ in the Riemannian models and the $n-1$-dimensional hyperboloid $H^{n-1} \simeq S O(n-1,1) / S O(n-1)$ in the Lorentzian models we consider here. The algebraic data $\left\{\rho_{f}\right\}$ and $\left\{x_{e}\right\}$ have a geometric interpretation in that the $\rho_{f}$ are to be thought of as volumes of the $(n-2)$-simplices dual to the faces of the 2-complex and the $x_{e}$ are to be thought of as unit normal vectors to the $(n-1)$-simplices dual to the edges of the 2-complex. While the expression for the edge amplitudes, interpreted as part of the gravitational measure together with the face amplitudes, varies in the various models, the vertex amplitude is the same in all these type of models and taken to be:

$$
\begin{array}{r}
\mathcal{A}_{v}\left(x_{e \in v}, \rho_{f \in v}\right)=\prod_{f \in v} \mathcal{A}_{f \in v}\left(x_{e 1 \in f}, x_{e 2 \in f}, \rho_{f}\right)= \\
=\prod_{f \in v} \mathcal{A}_{f \in v}\left(\theta_{f}, \rho_{f}\right)=\prod_{f \in v} D_{00}^{\rho_{f}}\left(\theta_{f}\right)
\end{array}
$$

i.e. they are given in terms of zonal spherical functions $D_{00}^{\rho_{f}}\left(\theta_{f}\right)$ of the Lorentz group in the representation $\rho_{f}$ [13], where we have indicated that the amplitudes depend on the variables on the homogeneous spaces only through the invariant distances $\theta_{f}=\cos (h)^{-1}\left(x_{e 1 \in f} \cdot x_{e 2 \in f}\right)$, between the vector associated to the two edges in the boundary of each face that are inside each vertex.

The structure is therefore that of a discrete path integral for gravity with a combinatorial structure playing the role of the base spacetime manifold, algebraic data living on it and playng the role of the gravitational degrees of freedom, and a (precisely defined) quantum amplitude and measure assigned to each configuration. In the group field theory approach to spin foam models (see [7, 8]), a sum over 2-complexes is also beautifully implemented. The issue now is to show that, on the one hand, it is possible even in this purely combinatorial/algebraic context to identify a notion of causal structure and appropriate data representing it, and then, on the other hand, that the spin foam formalism is flexible enough to provide us with a definition of both causal and a-causal transition amplitudes for quantum gravity, as the usual path integral formalism does for quantum particle dynamics or quantum field theory.

\section{Causality as orientation in spin foam models}

As we said, in spin foam models spacetime is replaced by a combinatorial 2-complex. Extra data would then assign geometric information to this spacetime structure. What can be 
the analogue of causal relations in such a context? Where is causality to be looked for? In the end the problem is solved more easily than could a priori be expected. Consider just the first layer of the spin foam 2-complex, i.e. only vertices and links connecting them. This is basically just a graph. If we add to it orientation data, i.e. arrows on the links, we obtain an oriented (or directed) graph, a set of oriented links connecting a set of vertices. Now the vertices can be interpreted as a set of fundamental spacetime events and the oriented links are then the causal relations between them (this is also consistent with an 'operational interpretation' of the dual simplicial structure, with $n$-simplices representing inperfect, thus realistic, definition of spacetime points/events). We can assign an orientation variable $\alpha_{e \mid v}$ to each link, with respect to each vertex $v$ it connects, that takes the values \pm 1 according to which orientation is chosen. This auotmatically defines 'oriented normals' $n_{e}=\alpha_{e \mid v} x_{e \mid v}$ from the un-oriented ones $x_{e}$. At the same time we can assign another orientation variable to each vertex, call it $\mu$ again taking the values \pm 1 . The spacetime interpretation of these variables is that of indicating whether the vertex is a future pointing or past pointing contribution to the overall spacetime diagram, and clearly a positively oriented link with respect to a future pointing vertex is equivalent to a negatively oriented one with respect to a past oriented vertex; this means that what gives the spacetime orientation of each link in each vertex is actually the combination $\alpha_{e \mid v} \mu_{v}$. A consistency condition for the assignment of orientation data to the graph is that when a link $e$ connects two vertices it has the opposite orientation in the two, which has a clear spacetime interpretation: $\alpha_{e \mid v_{1}} \mu_{v_{1}}=-\alpha_{e \mid v_{2}} \mu_{v_{2}}$. Taking now into account the full combinatorial structure of the 2-complex, we also assign an extra orientation variable to each face $\epsilon_{f}= \pm 1$. It is crucial to notice that the structure we have been describing is basically that of the Hesse diagram representing a causal set, but fails to be that of a proper poset or causal set [4] because the set of vertices-events endowed with the ordering relation represented by the arrow fails to satisfy in general any antisymmetry condition, i.e. it is generally not the case that following the links according to their orientation we never end up at the starting point; in other words, our causal relations allow for closed timelike loops. Also, notice that this causal interpretation of our oriented (or directed) graph makes sense only in a Lorentzian context, when the signature allows for a spacetime translation in terms of lightcones; however, the structure we have been describing remains the same even if thought of in a Riemannian signa- ture, and in fact the issue we will be confronting in the following is the general one of constructing spin foam models that reflect and take into account appropriately the orientation of the underlying 2-complex, i.e. of orientation-dependent transition amplitudes for quantum gravity. Only in a Lorentzian context these will have the interpretation of causal amplitudes or of quantum gravity analogues of the Feynman propagator. The 2-complexes used in spin foam models are not generic: they are topologically dual to simplicial n-dimensional manifolds: to each vertex corresponds a n-simplex, to each link a (n-1)-dimensional simplex, to each face an (n-2)-dimensional simplex; note that this gives another restriction with respect to a generic causal set. The orientation data we assigned have then a clear geometric interpretation in this simplicial picture: the $\mu_{v}$ variable for a vertex takes the values \pm 1 according to whether the n-simplex dual to it is isomorphic to a n-simplex in Minkowski (Euclidean) space or the isomorphism holds for the opposite orientation; the variable $\alpha_{e \mid v}= \pm 1$ indicate whether the normal to the (n-1)-simplex dual to the link $e$ is ingoing or outgoing with respect to the n-simplex dual to $v$, and the variables $\epsilon_{f}$ also characterize the orientation of the $n-2$-simplex dual to the face $f$. Knowing this dual geometric interpretation of the elements of the 2-complex, it is easy to derive a consistency condition on the values that these orientation variable must take to correspond to a well-posed orientation of the 2-complex (simplicial manifold); the relation, that basically follows from Stokes's theorem [12] is:

$$
\forall v \epsilon_{f \mid v}=\alpha_{e_{1} \mid v} \alpha_{e_{2} \mid v} \mu_{v}
$$

where $e_{1}$ and $e_{2}$ label the two links that belong to the boundary of the face $f$ and touch the vertex $v$.

This orientation structure is what we can identify at the quantum level as the seed for the emergence of causality in the classical limit, in a Lorentzian context, as we said; now the question is: do current spin foam model take this into account in their amplitudes? The answer $[1,2]$ is that all current spin foam models do not depend, in their amplitudes, on the orientation of the underlying 2-complex, i.e. they do not depend on the orientation data we identified above.

The way this is achieved is quite simple in all models: in the expression for the amplitudes for spin foams the terms that can be understood as contributions from opposite orientations are summed simmetrically thus erasing the dependence on the orientation itself. In the Barrett-Crane type of models, for example, the orientation independence is achieved at the level of each face in each vertex:

$$
\begin{aligned}
\mathcal{A}_{v}\left(x_{e \in v}, \rho_{f \in v}\right)=\prod_{f \in v} \mathcal{A}_{f \in v}\left(\theta_{f}, \rho_{f}\right)= \\
=\prod_{f \in v}\left(\mathcal{W}_{f}^{\epsilon_{f}=+1}\left(\theta_{f}, \rho_{f}\right)+\mathcal{W}_{f}^{\epsilon_{f}=-1}\left(\theta_{f}, \rho_{f}\right)\right)= \\
=\prod_{f \in v}\left(\mathcal{W}_{f}^{\mu_{v}=+1}\left(\alpha_{e 1} \alpha_{e 2} \theta_{f}, \rho_{f}\right)+\mathcal{W}_{f}^{\mu_{v}=-1}\left(\alpha_{e 1} \alpha_{e 2} \theta_{f}, \rho_{f}\right)\right)
\end{aligned}
$$


where we have indicated that the amplitudes depend on the variables on the homogeneous spaces only through the invariant distances $\theta_{f}=\cos (h)^{-1}\left(x_{e 1 \in f} \cdot x_{e 2 \in f}\right)$, between the vector associated to the two edges in the boundary of each face that are inside each vertex, and in the last step we have traded the orientation data on the faces for those on the vertices by using relation 4 . The mathematics behind this structure and the nature of the orientation-dependent functions $\mathcal{W}$ is very general, i.e. independent of signature and dimension, and it is explained in $[1,2]$.

This orientation independence leads to interpreting the current spin foam models as a-causal transition amplitudes, as we said, i.e. as the quantum gravity analogue of the Hadamard function of field theory and particle dynamics; when a canonical formulation is available, this sort of transition amplitudes can be equivalently thought of as defining the physical inner product between quantum gravity states, invariant under spacetime diffeomorphisms, or as a covariant definition of the matrix elements of the projector operator onto physical states [14].

Given the universal structure outlined above, we are lead to look for a universal way of modifying current spin foam models and to a new, again universal, definition of orientation-dependent or causal spin foam models [2]; these would correspond to causal transition amplitudes and to a quantum gravity analogue of the Feynman propagator of field theory. The "brute force"way is obvious and was performed in the 4-dimensional Lorentzian case in [12]: 1) Impose consistency conditions on orientation parameters $\epsilon_{f}, \alpha_{e}$ and $\mu_{v}$; 2) choose the orientation of simplices, in particular the value of $\mu_{v}$ (future or past-pointing), 3) restrict the spin foam amplitudes to include only the chosen orientation, i.e. switch from $\mathrm{D}$ functions to $W$ functions dropping the sum over $\mu_{v}$ or $\epsilon_{f}$. Hovewer, we will shortly see that a much more natural and elegant construction exists, that makes use of a generalised formulation of spin foam models in terms of a new proper time variable, from which all the different types of transition amplitudes can be defined, just as in the particle/QFT case. Also, the expression 5 is reminescent of the decomposition of Hadamard functions for relativistic particles or fields into Wightman functions, in turn the basic elements in the definition of the Feynman propagator. The construction we are going to describe now takes this analogy seriously and shows that it is indeed exact, and uses it as the staring point for a spin foam definition of the quantum gravity Feynman propagator that implements a notion of 'timeless ordering'.

\section{Particles on Lie groups/homogeneous spaces}

The amplitudes assigned to spin foam faces, edges and vertices in the Barrett-Crane-type models is given by the evaluation of simple spin networks and was described in [15] in analogy to the evaluation of Feynman diagrams: 1) assign a variable valued in the relevant homogeneous space to each vertex of the given spin network, 2) assign a zonal spherical function $D_{00}^{\rho_{f}}\left(\theta_{f}\right)$ to each line and 3) sum over all the possible values of the variables on the vertices to get the final amplitude. In this prescription the zonal spherical function is treated as a kind of propagator, and indeed on the one hand it turns out that this feynmanology has its roots in a (group) field theory of these models [7], and, on the other hand, it can be shown $[1,2]$ that the zonal spherical function used is indeed the Hadamard function for a scalar particle on the homogeneous space on which the models are based. This fact prompts a completely general definition of generalised, first, and then causal spin foam models.

Consider a scalar field $\phi(g)$ with mass $m$ living on the Lie group $G$ or on the homogeneous space $H$, with each point on it labelled by $g$; consider its free evolution parametrised by a proper time coordinate $s$; the equation of motion in proper time is: $\left(i \partial_{s}+\Delta\right) \phi(g, s)=0$ with $\Delta$ being the LaplaceBeltrami operator $G(H)$. The dynamics is completely captured by the evolution kernel $K\left(g, g^{\prime}, s\right)=K\left(g g^{-1}, s\right)$ [16], in the sense that given the initial condition $\psi\left(g_{0}, 0\right)$, we have: $\psi(g, s)=\int d g_{0} K\left(g, g_{0}, s\right) \psi\left(g_{0}, 0\right)$. The dependence on the proper time variable should of course be eliminated and the various physical propagators are obtained from the evolution kernel according to how this is accomplished; the Hadamard function is obtained via the expression: $H\left(g, g^{\prime}, m^{2}\right)=$ $-i \int_{-\infty}^{+\infty} d s K\left(g, g^{\prime}, s\right) e^{-i m^{2} s}$, while restricting the range of integration to positive proper times only gives the feynman propagator $G_{F}\left(g, g^{\prime}, m^{2}\right)=-i \int_{0}^{+\infty} d s K\left(g, g^{\prime}, s\right) e^{-i m^{2} s}$, where the usual Feynman prescription for the contour of integration $\left(m^{2} \rightarrow m^{2}-i \epsilon\right)$ is assumed for reasons of convergence (one may look at the two expressions as resulting from either a Fourier or a Laplace transform of the same function of proper time [2]).

It turns out [2] that the functions entering the expressions for the quantum amplitudes of all current spin foam models correspond to the Hadamard 2-point functions for a scalar field on the relevant Lie group/homogeneous space with $m^{2}=-C\left(\rho_{f}\right)$ where $C\left(\rho_{f}\right)$ is the Casimir eigenvalue of the simple irreducible representation labelling the face of the 2-complex (therefore the link of the spin network whose evaluation gives the amplitude for the vertex):

$$
\begin{gathered}
H\left(\theta_{f}, m^{2}\right)=-i \int_{-\infty}^{+\infty} d s K_{\mathcal{M}}\left(\theta_{f}, \mu_{v} s\right) e^{+i C\left(\rho_{f}\right) \mu_{v} s}= \\
=\frac{i}{2 \pi} \sqrt{\Delta_{\rho_{f} \in v}} D_{00}^{\rho_{f} \in v}\left(\theta_{f}\right)
\end{gathered}
$$

where the relevant orientation data $\mu_{v}$ enter in the definition of the proper time variable being integrated over, $\Delta_{\rho}$ is the dimension of the representation $\rho$ in the Riemannian case, or in the Lorentzian case (where the unitary representations are infinite dimensional) the contribution of the representation to the Plancherel measure, and the Casimir eigenvalues are $C(\rho)=2 j(2 j+n-2)$ with $j$ half-integer in the Riemannian case, and $C(\rho)=+\rho^{2}+(n-2 / 2)^{2}$ with $\rho$ positive real in the Lorentzian case based on the timelike hyperboloid. It is clear that the result is independent of the value of the various orientation data. 
On the other hand simply imposing the above restriction in the proper time integration (that amount indeed to a causality restriction for the particle evolution [5]), one obtains the expression for the Feynman propagator that one needs to define orientation dependent or causal spin foam models, that turns out [2] to have the expected expression in terms of $W$ functions confirming the interpretation of these as Wightman functions:

$$
\begin{aligned}
& G\left(\theta_{f}, m^{2}, \mu_{v}\right)=-i \int_{0}^{+\infty} d s K_{\mathcal{M}}\left(\vartheta_{f}, T\right) e^{+i C\left(\rho_{f}\right) T}= \\
& \quad=\frac{i}{2 \pi} \sqrt{\Delta_{\rho_{f}}}\left[\theta\left(\mu_{v}\right) \mathcal{W}^{+}\left(\vartheta_{f}, \rho_{f}\right)+\theta\left(-\mu_{v}\right) \mathcal{W}^{-}\left(\vartheta_{f}, \rho_{f}\right)\right]
\end{aligned}
$$

where we have denoted with $\vartheta$ the "oriented angle" $\vartheta_{f}=$ $\alpha_{e 1 \in f} \alpha_{e 2 \in f} \theta_{f}$, and with the "oriented proper time"being $T=\mu_{v} s$. Similarly for the other spin foam models [2].

This is a non-trivial function of the orientation data, with the usual "time ordering" being replaced by a "timeless ordering"!

\section{A proper time formalism for spin foam models and the spin foam de- finition of the Feynman propagator for quantum gravity}

Taking seriously this particle analogy and the associated spin network feynmanology, having also in mind their group field theory formulation [7], we can generalise the current formulation of spin foam models to include a proper time variable, and obtaining a general expression from which different transition amplitudes and consequently both orientation independent and causal models can be derived, simply changing the integration contour in proper time. The formulae have of course to include an explicit dependence on the orientation data. The expression for the $\mathrm{n}$-dimensional spin foam models $(n>3)$ based on the homogeneous space $\mathcal{M}$ looks as follows:

$$
Z=\left(\prod_{v} \prod_{f \mid v} \int_{C} d s_{f}\right)\left(\prod_{f} \sum_{\rho_{f}}\right)\left(\prod_{v} \prod_{e \mid v} \int d x_{e}\right) \mathcal{A}(\rho, x, T)
$$$$
\mathcal{A}(\rho, x, T)=\prod_{f} \mathcal{A}_{f}\left(\rho_{f}\right) \prod_{e} \mathcal{A}_{e}\left(\rho_{f \mid e}\right)
$$$$
\prod_{v} \prod_{f \mid v}\left(-2 \pi i\left(\sqrt{\Delta_{\rho_{f}}}\right)^{-1} K\left(\vartheta_{f \mid v}, T_{f \mid v}\right) e^{i C\left(\rho_{f}\right) T}\right) \text {. }
$$

A similar formula holds for the 3-dimensional models. This generalised expression encompasses both the usual unoriented models and the new causal ones; indeed the first are obtained by choosing the extended range of integration $C=(-\infty,+\infty)$ for the $s$ variable (and this erases the dependence on $\mu_{v}$ and $\alpha_{e}$ ), while the quantum gravity Feynman propagator is obtained with $C=(0,+\infty)$. In this last case, a regularization prescription for convergence is implicit for each variable (so that the expression has to be understood in the complex domain): $\rho_{f} \rightarrow \rho_{f}+i \epsilon, \vartheta_{f} \rightarrow \vartheta_{f}+i \delta$. The explicit form of the evolution kernel $K$ differs of course in the different models and affects the exact form of the amplitudes [2], that however all share the general structure here presented.

Notice that we have not modified the face and edge amplitudes with respect to the usual models. We could have done it: these amplitudes admit a Feynman graph-like evaluation as well and the technology related to quantum particles on homogeneous spaces could have been used to generalise and then modify them as we have done for the vertex amplitudes. The reason why we have not done so is twofold: on the one hand the usual interpretation of these contributions to the spin foam models is that of a conribution to the overall measure, therefore the implementation of causality is needed only at the vertex level, that is instead supposed to encode the dynamics of the theory; on the other hand, the form of the edge amplitudes in some version of the 4-d Barrett-Crane model is understood as arising directly from the form of boundary spin network states [17], and we prefer, at this stage of development of the theory, to keep that structure without modification. We believe, however, that alternative formulations of the models and possible definitions of modified spin network states, maybe in order to induce an orientatation-dependence in their structure, deserve further analysis.

Let us discuss the various properties of the new kind of transition amplitudes for quantum gravity we are defining here, the causal or orientation dependent spin foam models.

The first property we would like to stress is the fact that all these models can be recast in the form of quantum causal histories models [18] as it was done for the 4-dimensional Lorentzian case in [12]; therefore they define highly nontrivial amplitudes for causal sets, if the combinatorial structure of the underlying 2-complex is such that it does not contain closed timelike loops, although they are ceratainly a restriction of the possible causal sets one can consider since the vertices are here restricted to be $(n+1)$-valent in $n$ dimensions.

Second, the quantum amplitudes defined by these models can be related very easily to classical simplicial gravity, of which they clearly represent a covariant quantization: while the un-oriented models can be related to the classical Regge action only in a asymptotic limit, when their vertex amplitudes result in being proportional to the cosine of it, here the connection with the Regge action is manifest; for example, in the 4-dimensional case the relevant evolution kernel has the form [16]:

$$
K(\vartheta, T)=\frac{1}{(4 \pi i T)^{3 / 2}}\left(\frac{\vartheta}{\sinh \vartheta}\right) e^{i \frac{\vartheta^{2}}{4 T}-i T}
$$

the causal vertex amplitude, after the (restricted) proper time integration is perfomed, takes the form:

$$
\begin{aligned}
\mathcal{A}_{v}^{C} & =\prod_{f \in v}\left(-2 \pi i\left(\rho_{f}\right)^{(-1)} \int_{0}^{+\infty} d s_{f} K\left(\vartheta_{f}, T\right) e^{i C\left(\rho_{f}\right) T}\right)= \\
& =-\left(\prod_{f} \frac{1}{\rho_{f} \sinh \vartheta_{f}}\right) e^{i \sum_{f \in v} \mu_{v} \rho_{f} \vartheta_{f}}
\end{aligned}
$$


and therefore the product over vertex amplitudes in the spin foam model gives

$$
\begin{aligned}
\prod_{v} \mathcal{A}_{v}^{C} & =\left(\prod_{v} \prod_{f \in v} \frac{1}{\rho_{f} \sinh \vartheta_{f}}\right) e^{i \sum_{f \in v} \rho_{f} \sum_{v} \mu_{v} \vartheta_{f \in v}}= \\
& =\left(\prod_{v} \prod_{f \in v} \frac{1}{\rho_{f} \sinh \vartheta_{f}}\right) e^{i S_{R}\left(\rho_{f}, \vartheta_{f}\right)}
\end{aligned}
$$

i.e. the exponential of the Regge action (in first order formalism [12]) for simplicial gravity, apart from an additional contribution to the overall measure, as one would expect from a sum-over-histories formulation of quantum gravity based on a simplicial discretization. The same can be shown to be true explicitely in the $4 \mathrm{~d}$ Riemannian case and in the 3dimensional case, while the proof for the higher dimensional models is made more complicated by the lack of a simple enough expression for the evolution kernel [2].

\section{Conclusions}

Let us summarise what we have presented. We have discussed in which sense causality may be thought of preexisting (!) time at the most fundamental level, and what notion of causality, interpreted as a 'seed' from which the usual continuum notion of causality in terms of lightcone structure will emerge in a semiclassical approximation, can instead replace it in deep quantum gravity regime; we have argued that such a fundamental notion of causality can be implemented most naturally in a sum-over-histories context; with the aim of implementing this idea in the spin foam context, we have linked the notion of causality with the orientation of the 2-complex on which spin foam models are based, and have identified the relevant data characterizing this orientation, and found out that all current spin foam models are orientation-independent, i.e. defining amplitudes that trivial functions of the orientation data; using the technology of evolution kernels for quantum fields/particles on Lie groups and homogeneous spaces, we have constructed a generalised version of spin foam models, introducing an extra variable with the interpretation of proper time; we have shown that different ranges of integration for this proper time variable lead to different classes of spin foam models: one corresponds to the usual ones, to be interpreted as the quantum gravity analogue of the Hadamard function of QFT or equivalenty in a canonical interpretation as a covariant definition of the inner product between quantum gravity states; the other is a new class of models (one example of which having been constructed earlier in [12], and corresponds to the quantum gravity analogue of the Feynman propagator in QFT, i.e. a causal transition amplitude, a non-trivial function of the orientation data, that implies a notion of "timeless ordering", based on purely group-theoretic methods, as needed in Quantum Gravity; we have shown how the causal model is manifestly related to simplicial gravity in the 4-dimensional Lorentzian case. All these results hold true in full generality, for the type of spin foam models considered, i.e. regardless of the spacetime dimension and signature.

In our opinion these results open quite a few lines of possible further research. The causal/oriented models seem to solve the issue of multiplicity of sectors present in all BFtype formulations of quantum gravity [7], with different sectors isomorphic to one another and related by a change of orientation, all summed over in the path integral quantization and thus interphering, leading to a discrepancy of these models with the straightforward quantization of GR; in the causal models it seems instead that a restriction to the GR sector is achieved, as testified also by the final expression for the vertex amplitudes in the 4-dimensional case shown above. This is likely to have important consequencies for the reconstruction of geometric quantities from the algebraic data, in particular for the computation of expectation values of 3- and 4-volume operators, that are expected to give different results in these new models as compared to those obtained in the literature [8].

The group field theory formulation of generalised and oriented models should then be studied and work on this is indeed in progress, as a natural step that would substantiate the particle picture extensively used in their construction, and also to furnish a more complete definition of the models, eliminating any dependence on a fixed spacetime triangulation or 2-complex. This may turn out to be useful also for defining a notion of "positive and negative energy"sectors, also at the level of spin network states, based on orientation/causal properties, in a timeless framework. Such a result would also enforce the opinion, that we share, that the group field theory is not an "auxiliary"formulation of spin foam models and of quantum gravity, but the most fundamental formulation of the theory, and as such should be the framework in which most problems that this approach still faces are best tackled, including the issue of semiclassical/continuum approximation.

The proper time formalism we have developed could find interesting applications in itself, with a first step being the study of the relationship between our group theoretic proper time parameter and the lapse function of canonical quantum gravity or the conformal factor of the covariant formulation, and therefore with the physical proper time for quantum gravity (both the lapse and the conformal factor or spacetime volume element have used in the literature as a proper time variable). Notice that, if such a relationship is found, not only we would have strenghtened the argument for the validity of our constuction and paved the way for further developments, but we would have also obtained, as a side result (!), the first definition to be found in the literature, to the best of our knowledge, of a proper time expression for the action of simplicial gravity, that reduces to the Regge action when the additional variable is integrated out.

Another application of the proper time formalism could be the issue of Wick rotation in spin foam models, and more gnerally in quantum gravity, since it seems to be the right parameter in which to analytically continue the amplitudes to define an "euclideanized"model. 
Also, the generalised (proper time dependent) formulation of spin foam models could represent a new starting point for bridging the gap between spin foam models and other approaches to quantum gravity in which proper time plays a significant role, as for example the Lorentzian dynamical triangulations, that have achieved recently important results concerning the issue of continuum limit. The causal models seem indeed to be the at the point of convergence of simplicial quantum gravity, dynamical triangulations and causal sets, in addition to canonical loop quantum gravity, and therefore represent the easiest context in which to analyse the relationships between all these approaches.

Finally, if the orientation-independent models can be understood as defining the matrix elements of the projector operator onto physical quantum gravity states, then an intriguing possibility is to interpret the new models as defining the matrix elements of an "evolution operator", whose property could be studied to understand for example whether a notion of unitary evolution is feasible in Quantum Gravity and the "scattering"between quantum gravity perturbations, in absence of an external time coordinate, but with a clearly identified notion of causality.

\section{Acknowledgments}

I would like to thank gratefully Etera Livine and Florian Girelli for discussions and encouragement, and the organizers and staff of the DICE 2004 Workshop for a stimulating and truly enjoyable meeting.

\section{References}

[1] D. Oriti, Phys. Rev. Lett. 94, 111301 (2005); gr-qc/0410134.
[2] D. Oriti, in preparation.

[3] C. Isham, gr-qc/9210011.

[4] R. Sorkin, gr-qc/0309009.

[5] C. Teitelboim, Phys. Rev. D25, 3159 (1982).

[6] J.J. Halliwell and M.E. Ortiz, Phys. Rev. D 48, 748-768 (1993), gr-qc/9211004.

[7] D. Oriti, Rept. Prog. Phys. 64, 1489 (2001), gr-qc/0106091.

[8] A. Perez, Class. Quant. Grav. 20, R43 (2003), gr-qc/0301113.

[9] D. Oriti and R.M. Williams, Phys. Rev. D 63, 024022 (2001), gr-qc/0010031.

[10] A. Perez and C. Rovelli, Phys. Rev. D 63, 041501 (2001), grqc/0009021.

[11] L. Freidel, Nucl. Phys. Proc. Suppl. 88, 237 (2000), grqc/0102098.

[12] E. R. Livine and D. Oriti, Nucl. Phys. B 663, 231 (2003); grqc/0210064.

[13] N. Y. Vilenkin and A. U. Klimyk, Representations of Lie Groups and Special Functions, Vol.2 (Kluwer, Dordrecht, 1993).

[14] C. Rovelli, Phys. Rev. D 59, 104015 (1999), gr-qc/9806121.

[15] L. Freidel and K. Krasnov, J. Math. Phys. 41, 1681 (2000), hep-th/9903192.

[16] R. Camporesi, Phys. Rept. 196, 1 (1990).

[17] D. Oriti, Phys. Lett. B 532, 363 (2002), gr-qc/0201077.

[18] F. Markopoulou, Class. Quant. Grav. 17, 2059 (2000), hepth/9904009. 